\title{
Effects of periodic forcing in chaotic scattering
}

\author{
Fernando Blesa, ${ }^{1, *}$ Jesús M. Seoane, ${ }^{2, \dagger}$ Roberto Barrio, ${ }^{3}$ and Miguel A. F. Sanjuán ${ }^{2}$ \\ ${ }^{1}$ Computational Dynamics Group, Departamento de Física Aplicada, IUMA, Universidad de Zaragoza, E-50009 Zaragoza, Spain \\ ${ }^{2}$ Nonlinear Dynamics, Chaos and Complex Systems Group, Departamento de Física, Universidad Rey Juan Carlos, \\ Tulipán s/n, 28933 Móstoles, Madrid, Spain \\ ${ }^{3}$ Computational Dynamics Group, Departamento de Matemática Aplicada and IUMA, Universidad de Zaragoza, E-50009 Zaragoza, Spain
}

(Received 6 February 2014; published 17 April 2014)

\begin{abstract}
The effects of a periodic forcing on chaotic scattering are relevant in certain situations of physical interest. We investigate the effects of the forcing amplitude and the external frequency in both the survival probability of the particles in the scattering region and the exit basins associated to phase space. We have found an exponential decay law for the survival probability of the particles in the scattering region. A resonant-like behavior is uncovered where the critical values of the frequencies $\omega \simeq 1$ and $\omega \simeq 2$ permit the particles to escape faster than for other different values. On the other hand, the computation of the exit basins in phase space reveals the existence of Wada basins depending of the frequency values. We provide some heuristic arguments that are in good agreement with the numerical results. Our results are expected to be relevant for physical phenomena such as the effect of companion galaxies, among others.
\end{abstract}

DOI: 10.1103/PhysRevE.89.042909

PACS number(s): 05.45.Ac, 05.45.Df, 05.45.Pq

\section{INTRODUCTION}

Chaotic scattering in open Hamiltonian systems has been an area of study in nonlinear dynamics, with applications in several fields in physics [1,2].

A commonly studied setting is the particle motion in a potential field consisting of a group of potential hills [3,4]. In general, there exists a region where interactions between scattering particles and the potential occur, whereas, outside the region, the potential is negligible so the particle motions are essentially free. This region is often called the scattering region [1,3-5]. For many potential functions of physical interest, the corresponding classical Hamilton's equations of motion are nonlinear, rendering possible transient chaotic dynamics in the scattering region. Since the system is open, the region necessarily possesses "holes" for particles to enter and to escape. That is, particles from far away can enter the scattering region through one of the holes, experience some sort of chaotic dynamics in the region due to the interaction with the potential, and then exit the region through the same or a different hole. Because of the chaotic dynamics in the scattering region, particles with slightly different initial conditions (e.g., initial positions and momenta) can experience different paths of motion in the region and, consequently, they can spend drastically different times in the region and may exit through different holes in completely different directions. The sensitive dependence on the initial conditions of the scattering trajectory outcome makes the scattering chaotic.

Most previous works have focused on purely conservative systems [1,3-5]. More recently, the effects of both weak dissipation and noise on chaotic scattering have been addressed [6-13]. Despite a large body of existing literature on chaotic scattering, the effect of a periodic forcing on some properties of the scattering dynamics has received relatively little attention.

\footnotetext{
*fblesa@unizar.es

†jesus.seoane@urjc.es
}

The physical implications of this kind of perturbation, such as in problems concerning the effect of companion galaxies, chaotic Hamiltonian pumps, and oscillations in chemical reactions [14-20], among others, have motivated us to investigate its effects in the paradigmatic Hénon-Heiles system.

In Hamiltonian systems, regular motions, i.e., motions on various Kol'mogorov-Arnol'd-Moser (KAM) tori [21], are also fundamental. Depending on whether there are KAM tori coexisting with chaotic saddles in phase space, chaotic scattering may be characterized as either hyperbolic or nonhyperbolic. In hyperbolic chaotic scattering, all the periodic orbits are unstable and there are no KAM tori in phase space. In this case, the particle decay law is exponential, whereas in the nonhyperbolic case the particle decay law is algebraic. This decay law is drastically altered by the presence of external perturbations as dissipation and noise $[6,10]$ becoming an exponential decay law like, as noted above, in the hyperbolic case.

In this paper, we investigate the effects of an external forcing on both the decay law in the scattering region and the exit basins associated to phase space. We find an exponential decay law for the survival probability of the particles in the scattering region where the external frequency plays a crucial role in escape from the scattering region. We uncover a resonantlike behavior between the characteristic time $\tau$ of the decay law and the value of the frequency of the external forcing $\omega$ becoming $\omega \simeq 1$, the main resonant frequency.

Furthermore, the computation of the exit basins in phase space shows complicated basin structures depending on the forcing amplitude and the frequency of the external forcing. Our results also show that Wada basins are common in phase space. The role of both the external frequency and the forcing amplitude are crucial for the unpredictable behavior of our system.

This paper is organized as follows. In Sec. II, we describe our prototype model, the Hénon-Heiles system. The computation and analysis of the Wada property of the exit basins in phase space is carried out in Sec. III. In Sec. IV, we study the decay law of the particles in the scattering region showing a resonantlike behavior between the characteristic time and the 
frequency of the external forcing. Heuristic arguments of the previous results are shown in Sec. V. A discussion and the main conclusions of this manuscript are presented in Sec. VI.

\section{MODEL DESCRIPTION}

We now consider a continuous-time system as a prototype model of chaotic scattering, the forced Hénon-Heiles system [20]. The unperturbed Hamiltonian version of the system was first proposed in 1964 to address the question of whether there exist more than two constants of motion in the dynamics of a galaxy model [22], which correspond to bounded and unbounded orbits. The Hamiltonian is given by

$$
H=\frac{1}{2}\left(\dot{x}^{2}+\dot{y}^{2}\right)+\frac{1}{2}\left(x^{2}+y^{2}\right)+x^{2} y-\frac{1}{3} y^{3},
$$

which defines the motion of a particle of unit mass under the two-dimensional potential $V(x, y)=$ $\frac{1}{2}\left(x^{2}+y^{2}\right)+x^{2} y-\frac{1}{3} y^{3}$. Depending on its energy, a particle can be trapped in a region near $(x, y)=(0,0)$ or escape to infinity. The escaping energy $E_{e}$ is equal to $1 / 6$, above which particles can escape [22-24].

The contour plot of the Hénon-Heiles system is depicted in Fig. 1, where we also show the three exits and the eight nonlinear normal modes $\Pi_{i}$. A basic property of the HénonHeiles system is the existence of a class of highly unstable periodic orbits for $E>E_{e}$, called the Lyapunov orbits [25], $L_{i}$, which live near the border of the scattering region. When a trajectory crosses one of these periodic orbits from inside, it scatters off to infinity. Using the notation of Ref. [26] we can describe the nonlinear normal modes in the following way: The projections of the $\Pi_{1,2,3}$ orbits lie on the gradient lines of the potential that passes through the origin, and they are directed towards exits 1,2 , or 3 . Therefore $\Pi_{1,2,3}$ orbits disappear at the escape energy, when the Lyapunov orbits appear. As the Hénon-Heiles system has the $D_{3}$ symmetry, the projections are symmetric with respect to the $D_{3}$ group. The $\Pi_{4,5,6}$ orbits start on the level curves at a point with a given energy $E$ and intersect the gradient line $\Pi_{i-3}$ perpendicularly. Finally, $\Pi_{7}$ is a periodic orbit whose trajectory is counterclockwise forming perpendicular intersections with all three gradient lines $\Pi_{1,2,3}$.
Due to the temporal symmetry, $\Pi_{8}$ coincides with $\Pi_{7}$, but it goes clockwise [27].

The Hénon-Heiles Hamiltonian has a very rich fractal structure in phase space becoming Wada basins for suitable energy values (see, for instance, Ref. [24]). Figure 2 shows, for the Hénon-Heiles system in the absence of forcing, such distinct sets of initial conditions where the initial $x$ coordinate of the particles is $x(0)=0$. Blue (dark gray), red (gray), and yellow (light gray) denote the set of initial conditions that escape through exits 1,2 , and 3, respectively, and green (lower) denotes the initial conditions that are trapped in the scattering region. Initial conditions in Figs. 2(a) and 2(b) are taken to maintain the symmetry of the problem [24], i.e., the scalar product of position and velocity is zero. In Figs. 2(c) and 2(d) the initial conditions are $x(0)=0$, while $\dot{x}(0)$ is obtained from the energy. Finally, in Fig. 2(e) we take $x(0)=\dot{y}(0)=0$. For energy values $E=0.20$ and $E=0.25$ we show in Figs. 2(a) and 2(b) the basins in the physical space $(x, y)$ and in Figs. 2(c) and 2(d) the basins in the phase space $(y, \dot{y})$. The vertical lines in Figs. 2(a) and 2(b) denote the sections illustrated in Fig. 2(e) $(x(0)=0)$. Figure 2(e) represents the $(y, E)$ diagram in which we can observe that $E_{e}=1 / 6$ is the threshold value for which the particles can escape.

Periodic forcing can be conveniently introduced in the model by adding terms of the form $A \sin (\omega t)$ in the equations of motion [20] as follows:

$$
\begin{aligned}
\ddot{x}+x+2 x y & =A_{x} \sin \left(\omega_{x} t\right), \\
\ddot{y}+y+x^{2}-y^{2} & =A_{y} \sin \left(\omega_{y} t\right),
\end{aligned}
$$

where for simulations convenience, we take $A_{x}=A_{y}=A$ and $\omega_{x}=\omega_{y}=\omega$

Figures 3(a)-3(d) show typical trajectories of the Hénon-Heiles system with initial conditions $\left(x_{0}, \dot{x}_{0}, y_{0}, \dot{y}_{0}\right)=$ $(0,0,0,0)$ and parameter values $A=0.2$ and $\omega=0, \omega=1$, $\omega=10$, and $\omega=0.1$, respectively. We clearly see the effects of the external frequency since the particle can be trapped or can escape according to different manners depending on $\omega$ values. We can observe that the forcing induces different kind of dynamics such as quasiperiodic orbits [cases (a) and (c)], rapid escapes [case (b)] and slower escapes [case (d)], among
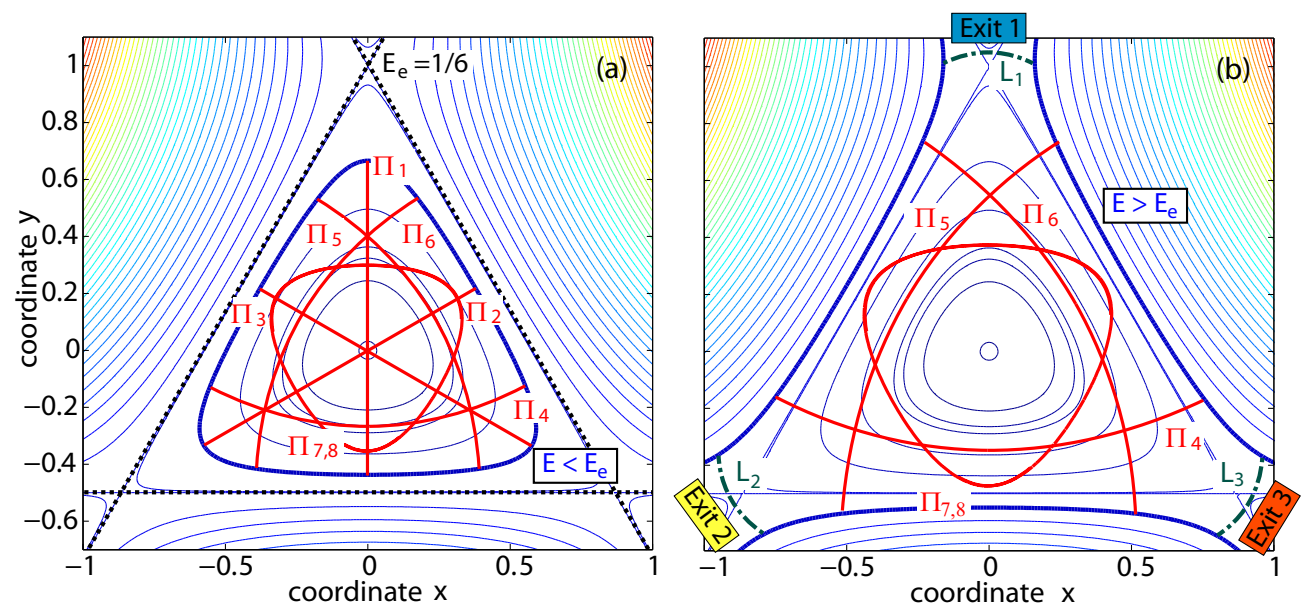

FIG. 1. (Color online) Level curves of the Hénon-Heiles potential, exits, limit of bounded motion, and the eight $\Pi_{i}$ nonlinear normal modes and three Lyapunov orbits $L_{i}$ on the (x,y) plane for (a) $E<E_{e}$ and (b) $E>E_{e}$. 

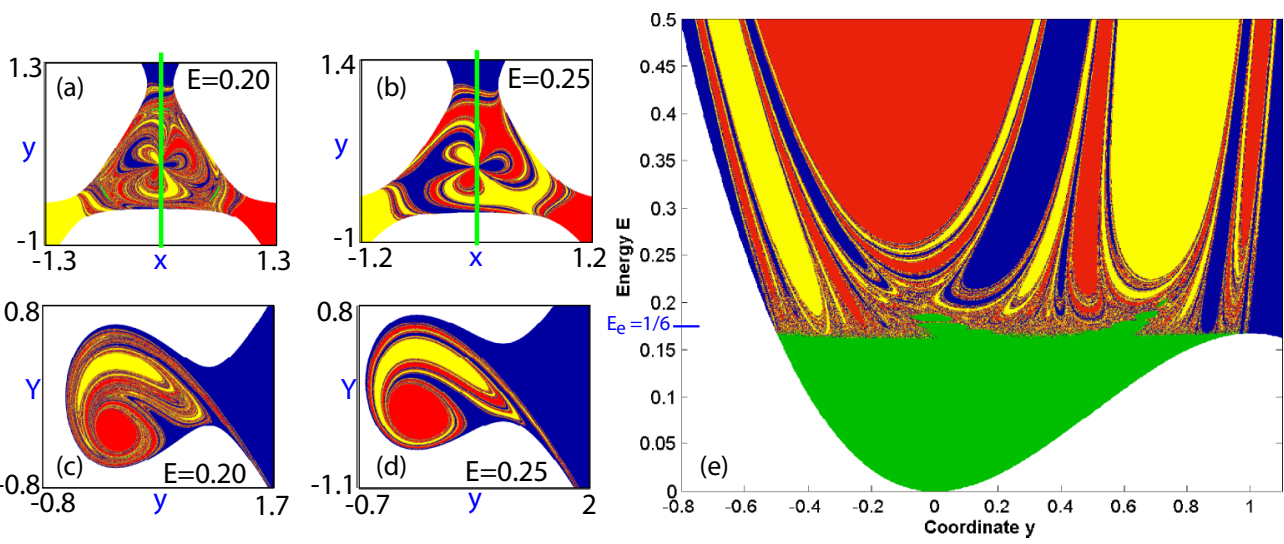

FIG. 2. (Color online) Plot of the exit basins of the unperturbed case. Blue (dark gray), red (gray), and yellow (light gray) denote the set of initial conditions that escape through exits 1,2, or 3, respectively. The green (lower) color on the right panel denotes the initial conditions that are trapped in the scattering region. Panels (a) and (b) [respectively, (c) and (d)] show the basins in physical space $(x, y)$ [in phase space $(y, \dot{y})]$ for two different values of the energy, $E=0.2$ and $E=0.25$. Panel (e) represents the $(y, E)$ diagram in which we can observe that $E_{e}=1 / 6$ is the threshold value for which the particles can escape. Initial conditions in panels (a) and (b) are chosen to maintain the symmetry of the problem. In panels (c) and (d) the initial conditions are such that $x(0)=0$ and $\dot{x}(0)$ is obtained from the energy $E$. Finally, in panel (e) we take $x(0)=\dot{y}(0)=0$, and $\dot{x}(0)$ is computed from $E$.

others. These different types of dynamics have motivated us to compute in the next section the exit basins in order to analyze the topology of the basins associated to phase space.

\section{BASIN STRUCTURE}

The exit basins associated to phase space in the HénonHeiles system can become very complicated in becoming the
Wada property [28]. In this section we show that a similar structure holds for the system with forcing in which, for that purpose, the value of the frequency plays a crucial role.

Mathematically, a basin is Wada if any boundary point also belongs to the boundaries of at least two other basins [29-31], i.e., every open neighborhood of a point belonging to a Wada basin boundary has a nonempty intersection with at least three different basins. If a dynamical system possesses
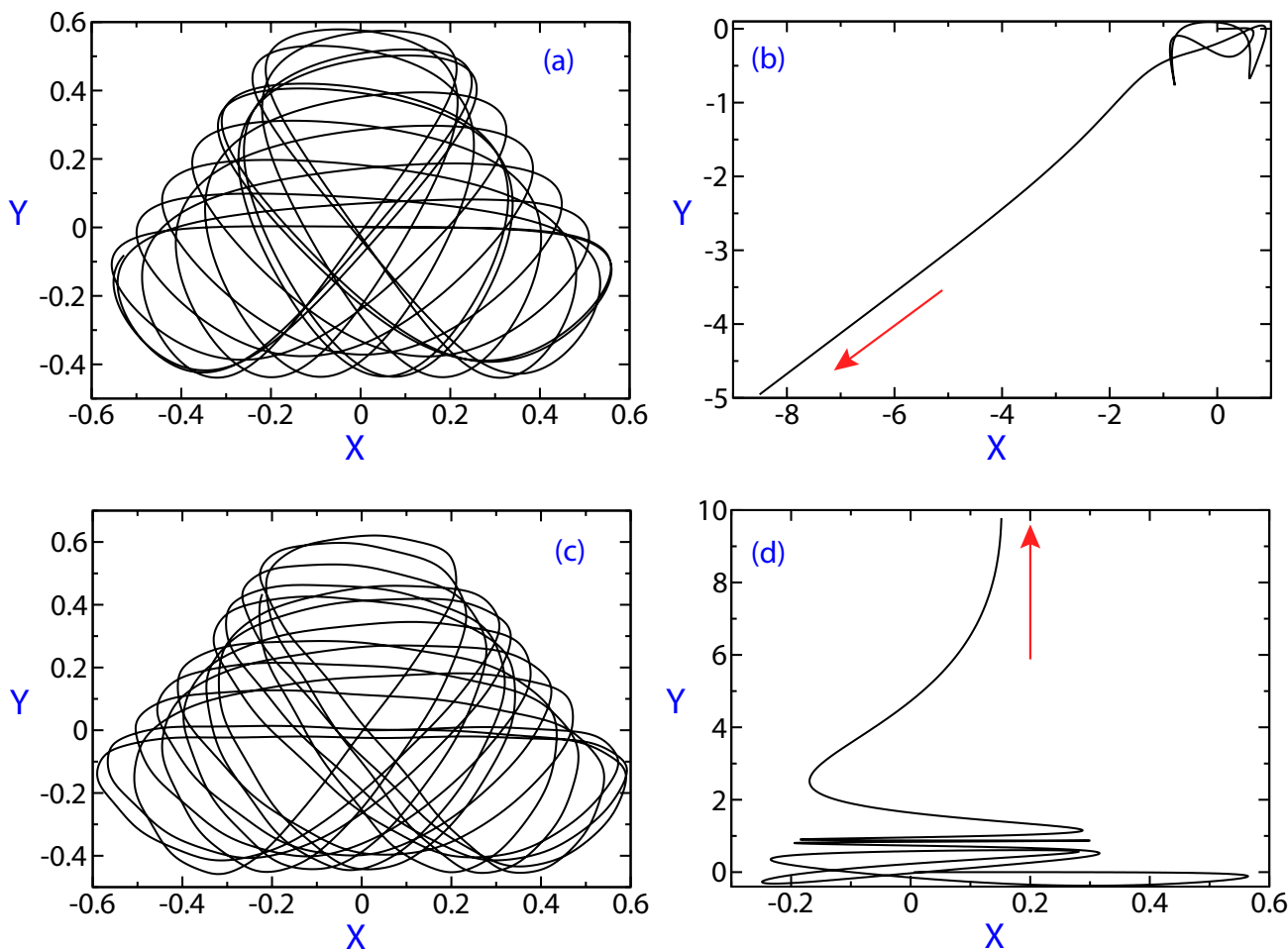

FIG. 3. (Color online) Typical trajectories of the forced Hénon-Heiles system with initial conditions $\left(x_{0}, \dot{x}_{0}, y_{0}, \dot{y}_{0}\right)=(0,0,0,0)$ and parameter values $A=0.2$ and (a) $\omega=0$, (b) $\omega=1$, (c) $\omega=10$, and (d) $\omega=0.1$, respectively. We can observe different dynamical behaviors depending on the frequency values: escaping dynamics [(b) and (c)] or quasiperiodic motions [(a) and (d)]. 

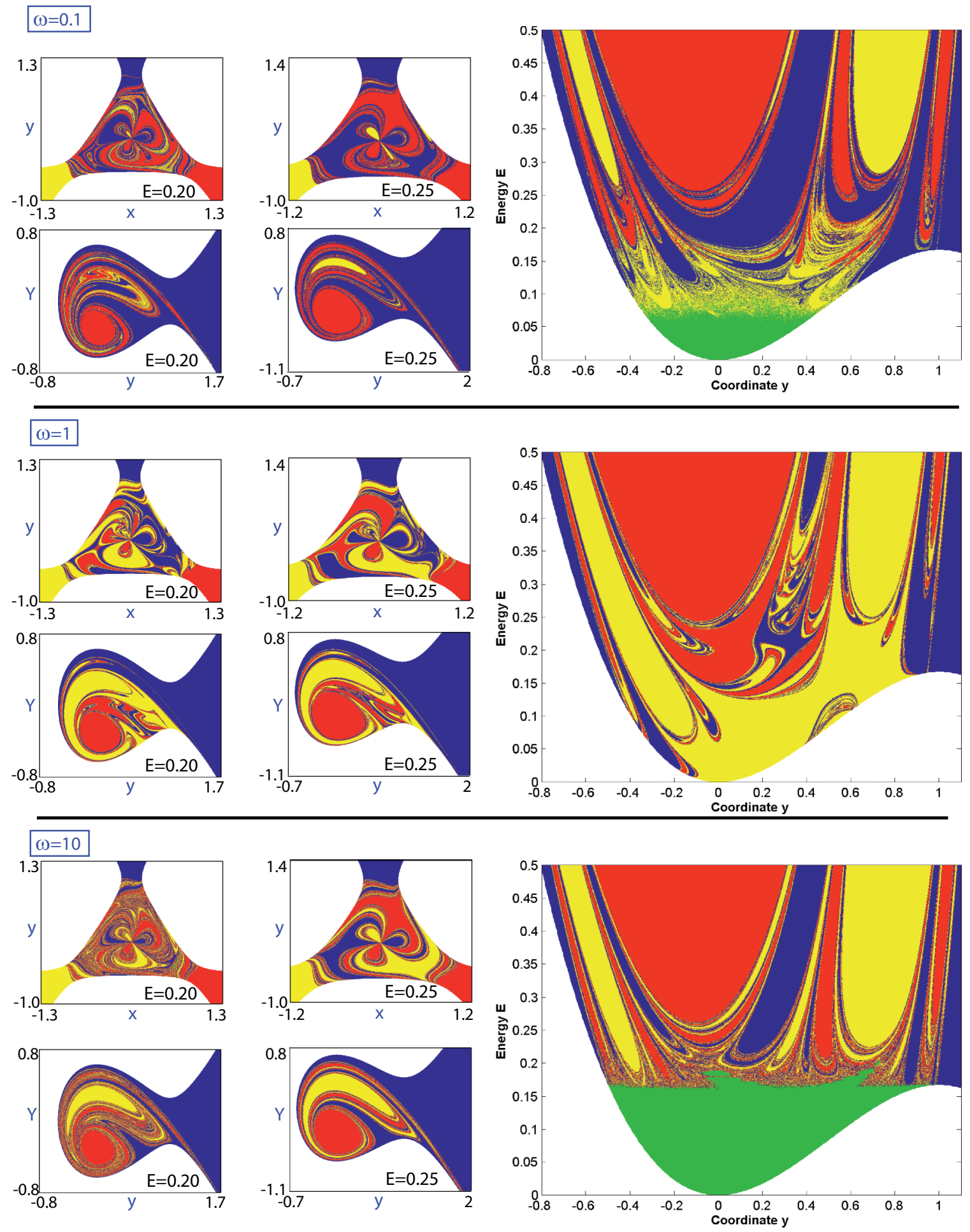

FIG. 4. (Color online) Plots of exit basins for the system with forcing with $\omega=0.1,1,10$. (Left) Plot of the exit basins and the basins of attraction of the Hénon-Heiles Hamiltonian in the physical space $(x, y)$ and the phase space $(y, \dot{y})$ for the energy values indicated in each figure. The color code is as follows: green (lower) corresponds to bounded orbits and blue (dark gray), yellow (light gray), and red (gray) correspond to the initial condition that leads to exits numbers 1,2 , and 3, respectively. (Right) Plot of the exit basins in the $(y, E)$ diagram. Initial conditions are chosen as in Fig. 2.

Wada basins, the degree of unpredictability of destinations can be more severe than the case where there are fractal basin boundaries with only two destinations [23,24,32].

In Figs. 4 and 5 the set of blue, red, and yellow dots denote initial conditions resulting in trajectories that escape through channels 1, 2, and 3 (Fig. 1), respectively. To generate the plots, a uniform grid of $500 \times 500$ initial conditions was chosen in the plotted region.

Figure 4 shows the exit basins, for the same simulation setting as Fig. 2, but with the system with forcing, with 

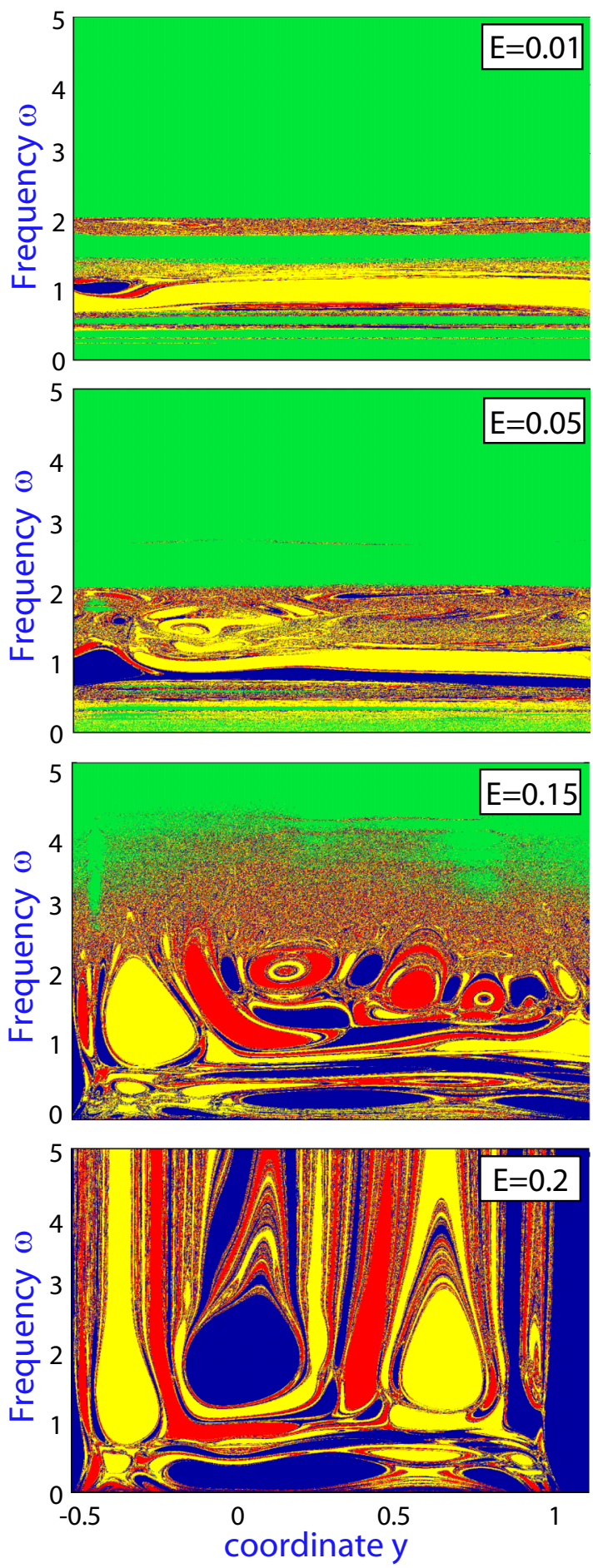

FIG. 5. (Color online) Plot of the $(y, \omega)$ diagram for $E=$ $0.01,0.05,0.15,0.2$. The color code is as described in the captions to previous figures. Green (upper) corresponds to bounded orbits. Notice that for $E=0.2$ there are escapes for all values of the frequency $\omega$ and resonant values also lead to escaping behavior in all the energies. Wada basins are present in presence of forcing as can be observed in the basins in both physical space $(x, y)$ and phase space $(y, Y)$ for $\omega=1, \omega=0,1$ and $\omega=10$.

frequency ( $\omega=0.1, \omega=1$, and $\omega=10$, respectively). The effects of the external frequency can be easily observed since the topology of the basins changes drastically. The

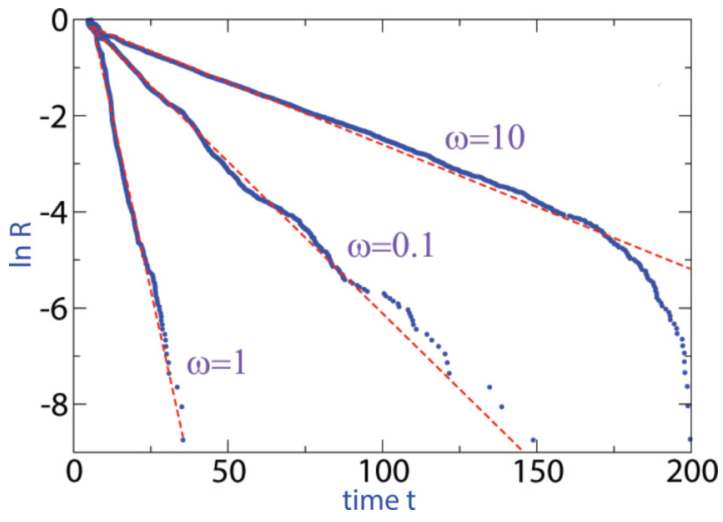

FIG. 6. (Color online) Typical exponential decay law for the particles remaining in the scattering region at time $t . R$ denotes the fraction of particles remaining in the scattering region. Here, we shoot $5 \times 10^{3}$ particles with energy $E=0.2$ from $\left(x_{0}, y_{0}\right)=(0,-0.5)$ and $\theta \in(0,2 \pi)$. The forcing amplitude is $A=0.1$. We observe the optimal value of the frequency $\omega=1$ for which the escapes take place faster than for other values of $\omega$.

left panels correspond to the physical space $(x, y)$ and phase space $(y, Y)$ for energies $E=0.20$ and $E=0.25$. We can observe in Fig. 4 that the basins in both physical space $(x, y)$ and phase space $(y, Y)$ for $\omega=1, \omega=0,1$ and $\omega=10$ have the Wada property. This result is due to the effect of the forcing since the forcing terms, amplitude $A$ and frequency $\omega$, introduce uncertainty in the evolution of the system and also induce transient chaos as typically occurs in chaotic scattering phenomena. The existence of Wada basins implies a high degree of unpredictability in the evolution of the system, which is produced by the existence of a forcing influence.

The right panels show the $(y, E)$ plane. From top to bottom, we show the evolution for several values of the frequency $\omega=$ $0.1,1,10$. The green region shows trapping dynamics since the energy is smaller than the threshold value and the frequency value is not the suitable one to help the particles escape from the scattering region (cases in which $\omega=0,1$ and $\omega=10$, where all particles escape, as shown in Fig. 6, after 150 and 200 time units, respectively). On the contrary, the value of $\omega=1$ produces the best performance for the escaping dynamics, as can be observed in the corresponding $(y, E)$ plane and also in Fig. 6, where all particles approximately escape after 30 time units. In this last case, all initial conditions escape from the scattering region as shown in Fig. 6. We uncover a resonant behavior that will be analyzed in the next section by studying the survival probability of the particles in the scattering region, obtaining the suitable values of $\omega$ for which escapes take place faster as compared with the conservative case.

Besides, for $\omega=1$ the basin structure is also very complicated and Wada basins appear.

On the other hand, in Fig. 4 we can observe that for high values of the frequency $\omega$, the basins are practically equivalent to the unperturbed system as a kind of averaging phenomena is performed. However, when $\omega$ is smaller, the situation differs. For the case $\omega=1$, we see that no matter the value of the energy there is no bounded motion. This case reveals that the resonant behavior plays a relevant role in the escape dynamics in periodic forced systems. When $\omega=0.1$, that is, small 
forcing values, the escape energy decreases compared with the unperturbed case, but the basins are similar to the unperturbed case.

If we vary $\omega$ while leaving the energy constant, we obtain the plots of Fig. 5. It shows the $(y, \omega)$ plots corresponding to several values of the energy: $E=0.01,0.05,0.15,0.2$. We can observe that for low and high values of the forcing frequency $\omega$ the motion is bounded if the energy values are small (below $E=0.1$ ). As the energy increases, the escape region grows, until it fills all the parameter space. We observe that the resonant values $\omega \simeq 1$ and $\omega \simeq 2$ give bands of escape behavior for any value of the energy. This is especially evident for low energies (for instance, the case of energy $E=0.01$ ), where, due to the resonant effect, we can see that the forcing can cause escapes, contrary to the unperturbed case that exhibits a bounded motion. This result is in good agreement with the previous one conjectured in the analysis of the basin structure in the planes $(x, y),(y, Y)$, and $(y, E)$ in which we observe faster escapes for $\omega=1$.

\section{DECAY LAW}

In nonhyperbolic chaotic scattering in Hamiltonian systems, the particle decay law is algebraic $[33,34]$. Perturbations as weak dissipation can turn KAM tori into periodic attractors [6]. As a result, the original nonattracting chaotic invariant set responsible for chaotic scattering undergoes a boundary crisis bifurcation to a set that generates fractal basin boundaries [35] typically arising in dissipative dynamical systems. The algebraic decay law in the conservative case will then become exponential in the dissipative case, no matter how weak the dissipation is. The same behavior takes place when noise is presented in a scattering system in which particles tend to escape faster from the scattering region as compared with the noiseless case. Therefore, we use the exponential decay law for the survival probability of the particles in the scattering region [10,11].

Insights into the switch in the decay law from algebraic to exponential can be gained by considering the classical model of a hierarchical construction of Cantor sets in the unit interval. First, an open subinterval in the middle of the unit interval is removed. Next, from each one of the remaining subintervals, the same fraction from the middle is removed, and so on. Each step of this scheme can be thought of as an iteration of the hyperbolic tent map with slope larger than 2 . The total length that remains decays exponentially with the number of iterations and the resulting Cantor set has a fractal dimension smaller than 1. This scenario corresponds to hyperbolic chaotic scattering.

For nonhyperbolic chaotic scattering, the same construction can be applied except that the fraction removed at each step decreases with time, e.g., is inversely proportional to time. This simple reduction of the fraction removed captures the essence of the effect of KAM tori [36]. The remaining length decays algebraically with time and, even though the measure of the remaining set approaches zero asymptotically, the resulting Cantor set has dimension 1 [36].

Due to the existence of dense orbits in the original chaotic set, the noncaptured part of the invariant set remains in the boundaries of the basins of the periodic attractors. Consequently, the invariant set is the asymptotic limit of the boundaries between scattered and captured orbits, rather than those between scattered and trapped orbits in the conservative case. Chaos occurs on the nonattracting invariant set whose stable manifold becomes the boundary separating the basins of the attractors and of the scattering trajectories. This reasoning suggests that the structure and the meaning of the Cantor set are altered by the effect of an external forcing. In particular, in successive steps we remove a constant instead of, in general, an increasing fraction in the middle of each interval, depending of both the amplitude of the external forcing and the frequency. The scattering dynamics becomes hyperbolic with exponential particle decay law.

The exponential-like decay law of the scattering particles is depicted in Fig. 6. In this picture, $R \sim \exp \gamma t$ denotes the fraction of particles remaining in the scattering region at time $t$. Here, we shoot $5 \times 10^{3}$ particles with energy $E=0.2$ from $\left(x_{0}, y_{0}\right)=(0,-0.5)$ and $\theta \in(0,2 \pi)$. The forcing amplitudes are $A=0.1$ and the forcing frequency is $\omega=1$ (resonant case), $\omega=0.1$ and $\omega=10$. The oscillations around the straight line obtained from the linear regression of the numerical data are due to the value of the chosen frequency $\omega$.

On the other hand, the numerical study of $\alpha=1 / \tau$ versus the logarithm of the external frequency $\omega$ for $E<E_{e}=0.15$ shows very clearly a resonant-like behavior in which the main resonant frequency is $\omega_{1} \simeq 1$, as shown in Fig. 7(a). The same results are shown in Fig. 7(b), for energy values $E>E_{e}=$ 0.20 , in which the same behavior can be observed. Other resonances are also evident (e.g., $\omega_{2} \simeq 2$, so $\ln \omega_{2} \simeq 0.69$ ).
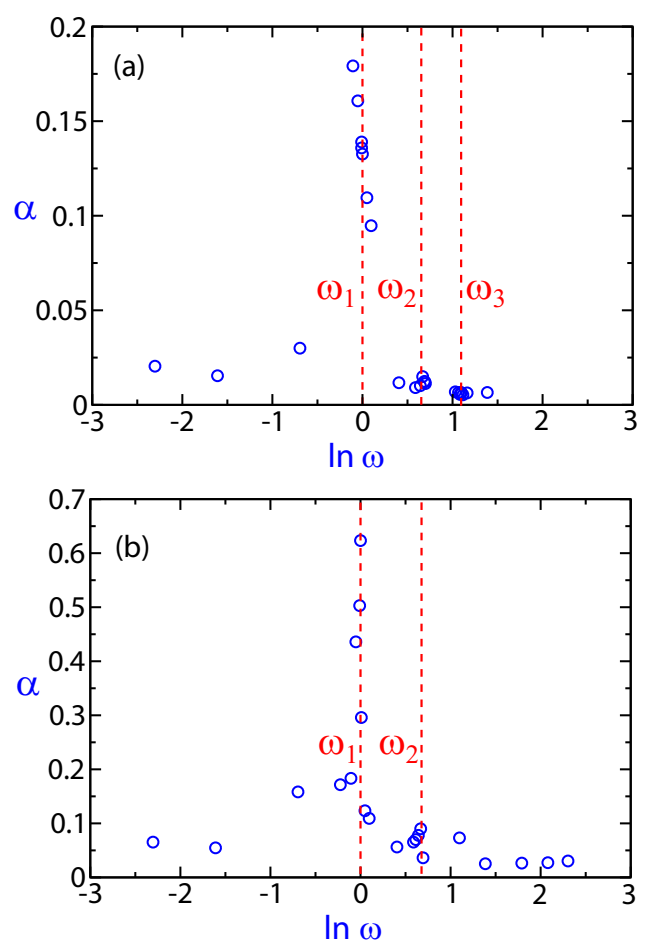

FIG. 7. (Color online) (a) Plot of $\alpha=1 / \tau$ versus $\ln$ of the external frequency $\ln (\omega)$, for $E=0.15<E_{e}$ and $A=0.1$, showing a resonant-like behavior in which the main resonant frequency is $\omega_{1} \simeq 1$. (b) A plot for the case $E=0.20>E_{e}$, where again this resonant phenomenon is observed very clearly. 


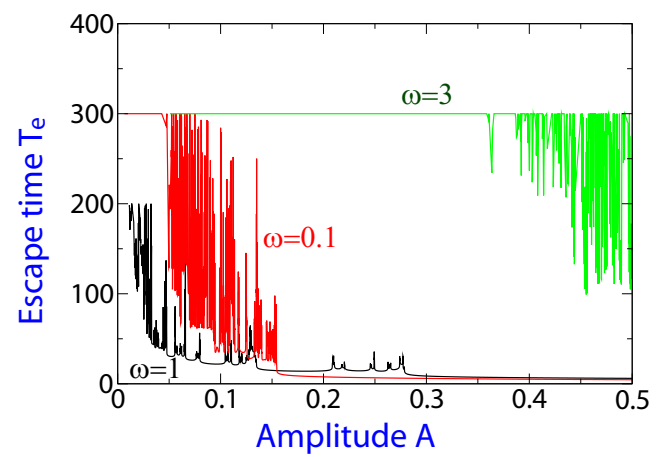

FIG. 8. (Color online) Plot of the escape times $T_{e}$ versus the amplitude $A$ of the external forcing, for a single trajectory, for $\omega=1$ (black), $\omega=10$ [green (light gray)], and $\omega=0.1$ [red (dark gray)], respectively. When the value of the amplitude of the external forcing is increased, the escape times of the particles decreases as expected. In all simulations $E=0.12, \theta=0$, and $\left(x_{0}, y_{0}\right)=(0,-0.5)$.

In order to provide more evidence on the resonant behavior, we analyze the escape times $T_{e}$ of the particles from the scattering region. For that purpose, first we study the escape time distributions versus the forcing amplitude $A$. We fix the value of the energy as $E=0.12$ and we use $\left(x_{0}, y_{0}\right)=(0,-0.5)$ as the initial condition. All the orbits are followed up to a maximum time 300 . Figure 8 represents, for a single trajectory with shooting angle $\theta=0$, the escape times $T_{e}$ versus the forcing amplitude for $\omega=1$ (black), $\omega=10$ [green (light gray)], and $\omega=0.1$ [red (gray)], respectively. When the value of the amplitude of the external forcing is increased, the escape times of the particles decrease for all cases, as expected.

To observe more clearly the influence of the resonances we show in Fig. 9 the plots of the escape times $T_{e}$ versus the frequency $\omega$ for several values of the energy up to a maximum time of 10000 . Each plot is obtained from the set of orbits $(500 \times 500$ orbits each) of the plots shown in Fig. 5. The red line shows the mean values of the escape times $T_{e}$ for the entire range of values for the $y$ coordinate. We plot the mean $\mu \pm 3 \sigma$ (standard deviation) in blue. Finally, in green we fill all the values inside the error. On one side, we should note that for $E=0.2$ all the orbits escape before we reach the maximum computation time, as expected, because $E$ is greater than the unperturbed escape energy $E_{e}$. On the other side, we can see that when the energy decreases, the escape time for frequencies $\omega \simeq 1$ and $\omega \simeq 2$ is much faster than for the rest of the frequencies (note that in the unperturbed case all these orbits are bounded). For low energies, when we are far from those resonant frequencies, we reach the maximum escape time without escaping, as expected since the energy in this case is small. Besides, we clearly observe that for large values of the forcing frequency $\omega$ the perturbation influence is averaged, meaning that its effect is not so relevant, and so the behavior is similar to that of the unperturbed case.

\section{HEURISTIC ARGUMENTS FOR THE FORCING EFFECTS}

As noted before, the unperturbed Hénon-Heiles potential has eight $\Pi_{i}$ nonlinear normal modes that have, at $E=0$, a
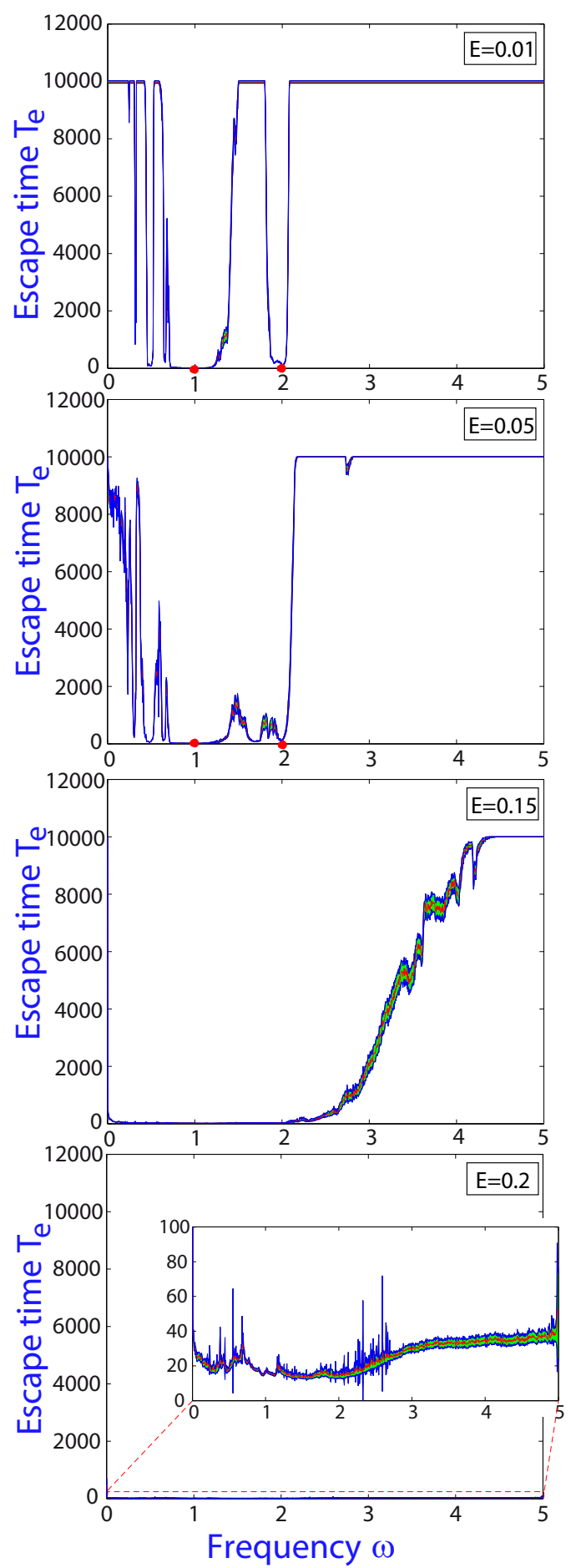

FIG. 9. (Color online) Plots of escape times $T_{e}$ vs frequency $\omega$ for several values of the energy up to a maximum propagation time of 10000 . From top to bottom: $E=0.01,0.05,0.15,0.2$. The mean values of the escape times for all values of coordinate $y$, as shown in Fig. 5, are depicted in red (gray). The mean plus-minus $3 \sigma$ error are depicted in blue (dark gray) and the values in between in green (light gray). Notice that for $E=0.2$ all the orbits escape before the maximum time. Forcing amplitude is $A=0.1$.

period $T_{i}=2 \pi$. This fact gives a main frequency $\omega=1$. Of course, when changing the energy, the period of the nonlinear modes changes as shown in Fig. 10. We can see that the ratio 


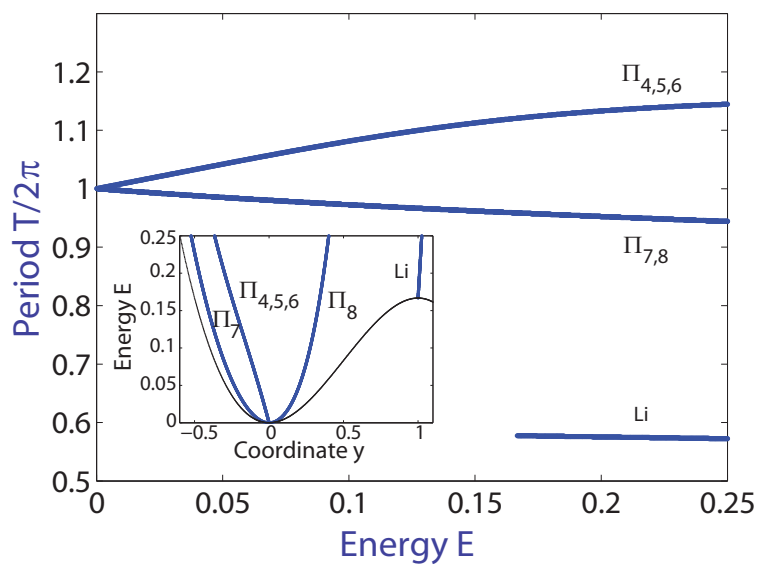

FIG. 10. (Color online) Plot of the orbit period $T_{i}$ versus the energy of the nonlinear normal modes $\Pi_{4-7}$ and the Lyapunov orbits $L_{i}$ for the unperturbed Hénon-Heiles Hamiltonian. Small inset: Plot of the nonlinear normal modes as the energy grows.

$T / 2 \pi$ is close to 1 for the normal modes. It differs in the case of the Lyapunov orbit that begins when the system is open. In the small inset we plot the main nonlinear normal modes in the plane $(y, E)$ (with $x=0$ ). After adding a periodic forcing term, when the frequency of the perturbation is resonant with the frequency of the unperturbed normal modes, an amplification phenomena will appear in the system, and this mechanism will help the orbit to escape (as shown in all the tests on this paper) because the energy is increased. That is, an average of the kinetic energy $K$ of the particles should present also local extrema for those values of the frequency. The reason is because as the kinetic energy, $K=\frac{1}{2}\left(\dot{x}^{2}+\dot{y}^{2}\right)$, increases, the velocity of the particles increases, too, and therefore the particles tend to escape from the scattering region very quickly as occurs for $\omega_{1} \simeq 1$ and $\omega_{2} \simeq 2$. For that purpose, we compute the kinetic energy for a propagation time corresponding to $3 T_{1} \simeq 3 \times 2 \pi$, in which $T_{1}$ is the period of the first normal mode.

Figure 11 shows the values of the average of the kinetic energy $K$ versus $\omega$ for an energy value $E=0.01$. We easily observe local maxima at $\omega_{1} \simeq 1$ and $\omega_{2} \simeq 2$, which correspond to the frequencies for which the particles escape from the scattering region very rapidly and agree with the resonant values. For larger values of $\omega$ an averaging process takes place and therefore the influence of the resonances decreases.

\section{CONCLUSION AND DISCUSSION}

In summary, our investigations on the effects of periodic forcing in the Hénon-Heiles Hamiltonian show an exponential

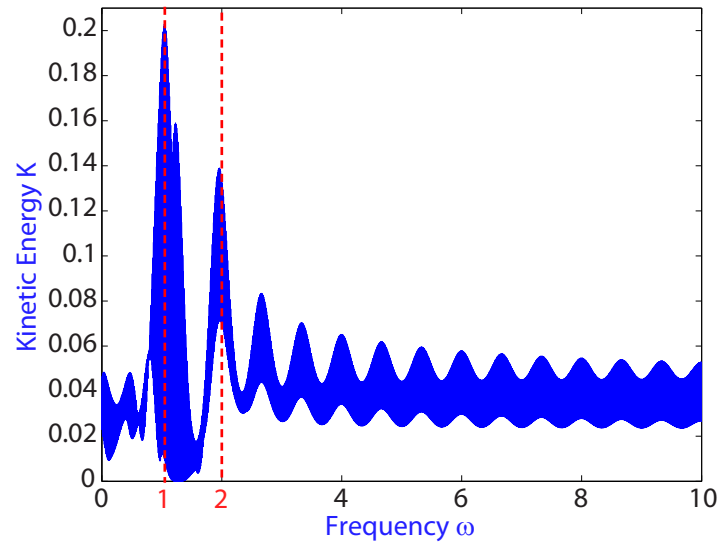

FIG. 11. (Color online) Plot of the final kinetic energy $K$ versus the frequency $\omega$ for $E=0.05$, forcing amplitude $A=0.1$, and propagation time $t=3 T_{1} \approx 3 \times 2 \pi$. We clearly observe the local extrema around the resonant frequencies $\omega \simeq 1$ and $\omega \simeq 2$, as previously mentioned.

decay law in the survival probability of the particles in the scattering region. The forcing term converts the system from nonhyperbolic to hyperbolic and the KAM islands in phase space disappear, therefore the decay turns exponential in contrast to the algebraic decay that is known from the autonomous system. Besides, the study of the characteristic time of the exponential decay law versus the frequency reveals a resonant-like behavior for frequency values $\omega_{1} \simeq 1$ and $\omega_{2} \simeq$ 2 for which the particles escape from the scattering region very quickly. These previous results are corroborated by computing the basins. This analysis also reveals the existence of Wada basins depending on the frequency values and therefore a high unpredictability in the evolution of the system.

A context of physical interest, where our result can be potentially useful, is in problems concerning the effects of companion galaxies, chaotic Hamiltonian pumps, and oscillations in chemical reactions, among others. We expect that this work can be useful for a better understanding of chaotic scattering phenomena in several physical contexts.

\section{ACKNOWLEDGMENTS}

This work was supported by the Spanish Ministry of Science and Innovation under Projects No. FIS2009-09898 (J.S. and M.S.) and No. MTM2012-31883 (R.B.). J.S. acknowledges the warm hospitality received at Universidad de Zaragoza, where part of this work was carried out.
[1] For a relatively early review of chaotic scattering, see: Chaos Focus Issue 3(4) (1993).

[2] J. M. Seoane and M. A. F. Sanjuán, Rep. Prog. Phys. 76, 016001 (2013).

[3] S. Bleher, C. Grebogi, and E. Ott, Physica D 46, 87 (1990).

[4] M. Ding, C. Grebogi, E. Ott, and J. A. Yorke, Phys. Rev. A 42, 7025 (1990).
[5] Some representative early papers on chaotic scattering are as follows: C. Jung, J. Phys. A 19, 1345 (1986); M. Hénon, Physica D 33, 132 (1988); P. Gaspard and S. A. Rice, J. Chem. Phys. 90, 2225 (1989); G. Troll and U. Smilansky, Physica D 35, 34 (1989).

[6] A. E. Motter and Y.-C. Lai, Phys. Rev. E 65, 015205(R) (2001). 
[7] J. M. Seoane, J. Aguirre, M. A. F. Sanjuán, and Y.-C. Lai, Chaos 16, 023101 (2006).

[8] J. M. Seoane, M. A. F. Sanjuán, and Y.-C. Lai, Phys. Rev. E 76, 016208 (2007).

[9] J. M. Seoane and M. A. F. Sanjuán, Int. J. Bifurcat. Chaos 20, 2783 (2010).

[10] J. M. Seoane and M. A. F. Sanjuán, Phys. Lett. A 372, 110 (2008).

[11] J. M. Seoane, L. Huang, M. A. F. Sanjuán, and Y. C. Lai, Phys. Rev. E 79, 047202 (2009).

[12] E. G. Altmann and A. Endler, Phys. Rev. Lett. 105, 244102 (2010).

[13] J. D. Bernal, J. M. Seoane, and M. A. F. Sanjuán, Phys. Rev. E 88, 032914 (2013).

[14] R. Ramaswamy, P. Siders, and R. A. Marcus, J. Chem. Phys. 74, 4418 (1981).

[15] H. E. Kandrup and S. J. Novotny, Cel. Mech. Dyn. Astr. 88, 1 (2004).

[16] S. Kawai, A. D. Bandrauk, C. Jaffé, T. Bartsch, J. Palacián, and T. Uzer, J. Chem. Phys. 126, 164306 (2007).

[17] D. Hennig, L. Schimansky-Geier, and P. Hänggi, Eur. Phys. J. B 62, 493 (2008).

[18] Q. Zhang, P. Hänggi, and J. Gong, New J. Phys. 10, 073008 (2008).

[19] T. Dittrich, M. Gutiérrez, and G. Sinuco, Physica A 327, 145 (2003).

[20] F. Blesa, J. M. Seoane, R. Barrio, and M. A. F. Sanjuán, Int. J. Bifurcat. Chaos 22, 1230010 (2012).

[21] J. Moser, Stable and Random Motions in Dynamical Systems (Princeton University Press, Princeton, NJ, 1973).
[22] M. Hénon and C. Heiles, Astron. J. 69, 73 (1964).

[23] L. Poon, J. Campos, E. Ott, and C. Grebogi, Int. J. Bifurcat. Chaos 6, 251 (1996).

[24] J. Aguirre, J. C. Vallejo, and M. A. F. Sanjuán, Phys. Rev. E 64, 066208 (2001).

[25] G. Contopoulos, Astron. Astrophys. 231, 41 (1990).

[26] R. C. Churchill, G. Pecelli, and D. L. Rod, Arch. Rat. Mech. Anal. 73, 313347 (1980).

[27] R. Barrio, F. Blesa, and S. Serrano, New J. Phys. 11, 053004 (2009).

[28] R. Barrio, F. Blesa, and S. Serrano, Europhys. Lett. 82, 10003 (2008).

[29] J. Kennedy and J. A. Yorke, Physica D 51, 213 (1991).

[30] Z. Toroczkai, G. Károlyi, A. Péntek, T. Tél, C. Grebogi, and J. A. Yorke, Physica A 239, 235 (1997); M. A. F. Sanjuán, J. Kennedy, C. Grebogi, and J. A. Yorke, Chaos 7, 125 (1997); J. Kennedy, M. A. F. Sanjuán, J. A. Yorke, and C. Grebogi, Topol. Appl. 94, 207 (1999).

[31] H. E. Nusse and J. A. Yorke, Science 271, 1376 (1996); Physica D 90, 242 (1996); Phys. Rev. Lett. 84, 626 (2000).

[32] J. Aguirre and M. A. F. Sanjuán, Physica D 171, 41 (2002).

[33] C. F. F. Karney, Physica D 8, 360 (1983).

[34] Y. C. Lai, M. Ding, C. Grebogi, and R. Blümel, Phys. Rev. A 46, 4661 (1992).

[35] S. W. McDonald, C. Grebogi, E. Ott, and J. A. Yorke, Physica D 17, 125 (1985).

[36] Y. T. Lau, J. M. Finn, and E. Ott, Phys. Rev. Lett. 66, 978 (1991). 